\title{
Circulating CD15+LOX-1+ PMN-MDSC is a potential biomarker for the recurrence after surgery of non-small cell lung cancer
}

\author{
Xinyu Tian ${ }^{1}$, Qisi Zheng ${ }^{1}$, Yue Tao ${ }^{1}$, Lei Dai ${ }^{1}$, Ting Wang $^{2}$, and Han Shen ${ }^{1}$ \\ ${ }^{1}$ Nanjing Drum Tower Hospital \\ ${ }^{2}$ Jiangsu Cancer Hospital
}

November 9, 2020

\begin{abstract}
Aims: As a population of immunosuppressive cells, polymorphonuclear-myeloid derived suppressor cells (PMN-MDSCs) are responsible for lung cancer metastasis, and metastasis is a main cause of the recurrence after surgery of non-small-cell lung cancer (NSCLC). Lectin-type oxidized LDL receptor 1 (LOX-1) is a newly confirmed maker for identifying PMN-MDSCs in human. In this study, we tried to confirm the relationship between the frequency of newly identified CD15+LOX-1+ PMN-MDSCs and NSCLC recurrence after surgery. Methods: Flow cytometry (FCM) was used to detect the proportion of CD15+LOX-1+ PMN-MDSCs in the peripheral blood (PB) cells of healthy controls (HC) and NSCLC patients. The correlation of CD15+LOX-1+ PMN-MDSC frequency with levels of cytokeratin 19-fragments (CYFRA21-1), carcinoembryonic antigen (CEA), and carbohydrate antigen 125 (CA125) was analyzed. Receiver operating characteristic (ROC) curve was used to estimate the diagnostic efficacy of CD15+LOX-1+ PMN-MDSC frequency for NSCLC. Additionally, the association of CD15+LOX-1+ PMN-MDSCs with NSCLC prognosis and recurrence after surgery was explored. Results: The proportion of CD15+LOX-1+ PMN-MDSCs increased significantly in PB of NSCLC patients. CD15+LOX-1+ PMN-MDSC proportion was positively correlated with levels of CEA and CYFRA21-1, but not CA125. The area under the ROC curve (AUC) of PMN-MDSC percentage was higher than CYFRA21-1, CEA and CA125. Compared to NSCLC patients before surgery, the proportion of CD15+LOX-1+ PMN-MDSCs decreased in patients after surgery. The frequency of CD15+LOX-1+ PMN-MDSCs was lower in NSCLC patients without recurrence compared to those with recurrence after surgery. Conclusions: Circulating CD15+LOX-1+ PMN-MDSCs are a potential diagnostic marker for NSCLC, and are associated with NSCLC recurrence after surgery.
\end{abstract}

Circulating $\mathrm{CD}^{+}{ }^{+} \mathrm{LOX}-1^{+} \mathrm{PMN}-\mathrm{MDSC}$ is a potential biomarker for the recurrence after surgery of non-small cell lung cancer

Xinyu Tian ${ }^{1 *}$, Qisi Zheng ${ }^{1 \#}$, Yue Tao ${ }^{1 \#}$, Lei Dai ${ }^{1}$, Ting Wang ${ }^{2 *}$, and Han Shen ${ }^{1 *}$

1 Department of Laboratory Medicine, Nanjing Drum Tower Hospital, Nanjing University Medical School, Nanjing, Jiangsu, China

2 Department of Laboratory Medicine, Jiangsu Cancer Hospital \& Jiangsu Institute of Cancer Research \& The Affiliated Cancer Hospital of Nanjing Medical University, Nanjing, Jiangsu, China

*Corresponding authors:

Xinyu Tian, Department of Laboratory Medicine, Nanjing Drum Tower Hospital, 321 Zhongshan Road, Nanjing 210008, China. Tel.: +86025 83106666; E-mail:1000004433@ujs.edu.cn

Ting Wang, Department of Laboratory Medicine, Jiangsu Cancer Hospital \& Jiangsu Institute of Cancer Research \& The Affiliated Cancer Hospital of Nanjing Medical University, 42 Baiziting Road, Nanjing 210009, China. Tel.: +86025 83283597; Fax: +86025 83283597; E-mail:jipinxinyu@163.com 
Han Shen, Department of Laboratory Medicine, Nanjing Drum Tower Hospital, 321 Zhongshan Road, Nanjing 210008, China. Tel.: +86 025 83106666; E-mail: shenhan10366@sina.com

\# The three authors contributed equally.

\section{DISCLOSURES:}

For each author there were no conflicts of interests, including all relevant financial interest in any company or institution that might benefit from the publication.

\section{ABSTRACT}

Aims: As a population of immunosuppressive cells, polymorphonuclear-myeloid derived suppressor cells (PMN-MDSCs) are responsible for lung cancer metastasis, and metastasis is a main cause of the recurrence after surgery of non-small-cell lung cancer (NSCLC). Lectin-type oxidized LDL receptor 1 (LOX-1) is a newly confirmed maker for identifying PMN-MDSCs in human. In this study, we tried to confirm the relationship between the frequency of newly identified CD15+LOX-1+ PMN-MDSCs and NSCLC recurrence after surgery.

Methods: Flow cytometry (FCM) was used to detect the proportion of CD15+LOX-1+ PMN-MDSCs in the peripheral blood (PB) cells of healthy controls (HC) and NSCLC patients. The correlation of CD15 ${ }^{+}$LOX$1^{+}$PMN-MDSC frequency with levels of cytokeratin 19-fragments (CYFRA21-1), carcinoembryonic antigen (CEA), and carbohydrate antigen 125 (CA125) was analyzed. Receiver operating characteristic (ROC) curve was used to estimate the diagnostic efficacy of CD15+LOX-1+ PMN-MDSC frequency for NSCLC. Additionally, the association of CD $15^{+} \mathrm{LOX}-1^{+}$PMN-MDSCs with NSCLC prognosis and recurrence after surgery was explored.

Results: The proportion of CD $15^{+} \mathrm{LOX}-1^{+}$PMN-MDSCs increased significantly in PB of NSCLC patients. CD $15^{+}$LOX-1 ${ }^{+}$PMN-MDSC proportion was positively correlated with levels of CEA and CYFRA21-1, but not CA125. The area under the ROC curve (AUC) of PMN-MDSC percentage was higher than CYFRA21-1, CEA and CA125. Compared to NSCLC patients before surgery, the proportion of CD15+LOX-1+ PMNMDSCs decreased in patients after surgery. The frequency of CD $15^{+}$LOX-1 $1^{+}$PMN-MDSCs was lower in NSCLC patients without recurrence compared to those with recurrence after surgery.

Conclusions: Circulating CD15 ${ }^{+} \mathrm{LOX}-1^{+}$PMN-MDSCs are a potential diagnostic marker for NSCLC, and are associated with NSCLC recurrence after surgery.

KEYWORDS: Polymorphonuclear-myeloid derived suppressor cells, non-small cell lung cancer, biomarker, diagnosis, recurrence after surgery

\section{WHAT IS KNOWN?}

1. PMN-MDSCs are involved in the progression of NSCLC by promoting tumor immune escape and metastasis.

2. Initially, PMN-MDSCs shared similar morphology and phenotype with mature neutrophils, and the suppressive activity was the most reliable approach to identify PMN-MDSCs.

3. LOX-1 is a newly confirmed maker for identifying PMN-MDSCs in human.

\section{WHAT IS NEW?}

1. $\mathrm{CD} 15^{+} \mathrm{LOX}-1^{+}$PMN-MDSC frequency is increased in PB cells of NSCLC patients, and is a potential biomarker for NSCLC diagnosis.

2. The proportion of $\mathrm{CD} 15^{+} \mathrm{LOX}-1^{+} \mathrm{PMN}-\mathrm{MDSC}$ is associated with NSCLC prognosis and recurrence after surgery.

\section{INTRODUCTION}


Lung cancer has become a challenging health problem worldwide. More than 1.0 million people die of the disease each year [1, 2]. According to the histopathological classification, lung cancer can be divided into small cell lung cancer (SCLC) and NSCLC, in which NSCLC accounts for approximately $85 \%$ and is the most common type of clinical lung cancer. Moreover, NSCLC can be subdivided into squamous cell carcinoma, adenocarcinoma and large cell carcinoma [3-5]. In spite of the development in lung cancer treatment, such as surgery combined with radiotherapy, chemotherapy, and immunotherapies, NSCLC recurrence after surgery still remains at a high rate [6-8]. Cancer metastasis is identified as the main cause of cancer related mortalities after resection. It is confirmed that $30-55 \%$ of NSCLC patients in early stage die from recurrent metastatic disease after surgery with curative intent, and only $3-10 \%$ of them can survive over 5 years with the treatment of standard adjuvant chemotherapy [9-11]. Immune checkpoint blockade that can promote the generation of antigen specific $\mathrm{T}$ cells is a newly defined immune adjuvant therapy $[12,13]$. However, most of early-stage NSCLC tumors are removed without the absence of neoadjuvant intervention in clinical practice [10, 14]. Therefore, novel strategies are necessary for monitoring and decreasing the recurrence and metastasis in patients with primary NSCLC.

In the progression of lung cancer, there exist multiple immune cells infiltrating into tumor sites. Different types of these immune cells and their products determine the fate of tumor progression. Immune cells also play a major role in establishing metastasis of the primary tumor to various organs [15-18]. A protective immune response against tumor is often prevailed by the protumor response, and it eventually is the balance or the lack of it between these two processes that determine the fate of tumor growth and metastasis. One of the immuosuppressive populations that are rapidly attracting attention in tumor biology is MDSCs [19]. MDSCs are a population of immature myeloid cells (IMCs) with immune suppressive activity. Under physiological conditions, IMCs produced by bone marrow can differentiate into mature granulocytes, monocytes and dendritic cells (DCs). In pathological conditions especially cancer, the differentiation of IMCs is inhibited by molecules released by tumor cells, stromal cells and activated immune cells, which leads to the accumulation of MDSCs [20-22]. In human and mice, PMN-MDSCs and monocytic MDSCs (M-MDSCs) are the two main subsets of MDSCs, in which PMN-MDSCs that account for $70-80 \%$ of total MDSCs are the prioritized population [23]. PMN-MDSCs have been confirmed to be involved in the progression of NSCLC by promoting tumor immune escape and metastasis in both preclinical and clinical trails [24-26]. However, PMN-MDSCs were initially identified as a population of terminally differentiated neutrophils with immunosuppression, which shared similar morphology and phenotype with mature ones. Therefore, it was the suppressive activity, but not phenotype, was the most reliable approach to distinguish PMN-MDSCs from neutrophils [19]. Recently, it was confirmed that LOX-1 could be used to distinguish the population of PMN-MDSCs in cancer patients [27-29]. Thus, in present study, we tried to ensure the application of newly defined CD15+LOX-1+ PMN-MDSCs in NSCLC diagnosis and prognosis, as well as identify their association with NSCLC recurrence after surgery.

\section{MATERIALS AND METHODS}

\section{Patients and samples}

PB samples of primary NSCLC patients (Adenocarcinoma, $n=27$; Squamous cell lung cancer, $n=23$ ) and healthy donors $(n=50)$ were obtained from Nanjing Drum Tower Hospital from April 2018 to December 2019. Cancer cases were confirmed by pathological diagnosis. According to the results of surgery, cancer staging was determined according to the 6th edition of the American Joint Cancer Commission's tumor-node-metastasis (TNM) staging system. All NSCLC patients were diagnosed for the first time and had not received any treatment before. Detailed information of NSCLC patients were listed in Table 1. Collected samples were firstly subjected to centrifuge at $20^{\circ} \mathrm{C}, 2000 \mathrm{rpm}$ for $5 \mathrm{~min}$ to separate the cells from plasma. ACK buffer was used to lyse red blood cells (RBCs), and the remaining cells were used for following experiments. To confirm the potential application of PMN-MDSC frequency in NSCLC diagnosis, we also collected paired PB samples from NSCLC patients at one month after surgical resection. Meanwhile, PB samples of NSCLC patients with or without recurrence after surgery were collected to identify the association of PMN-MDSC proportion with NSCLC recurrence $(\mathrm{n}=50)$. All samples were obtained in accordance with the approval of the 
Ethics Committee of Nanjing Drum Tower Hospital. All experiments were undertaken with the understanding and written consent of each subject, and this study conforms with The Code of Ethics of the World Medical Association (Declaration of Helsinki), printed in the British Medical Journal (18 July 1964).

\section{FCM}

To detect the proportion of CD15+LOX-1+ PMN-MDSCs in PB of NSCLC patients and HC, ACK buffer was used to lyse RBCs, and the remaining cells were stained with PE-anti-human-LOX-1 (eBioscience, $0.25 \mu \mathrm{g} / 1 \times 10^{6}$ cells) and FITC-anti-human-CD15 mAbs (eBioscience, $0.25 \mu \mathrm{g} / 1 \times 10^{6}$ cells) at $4^{\mathrm{O}} \mathrm{C}$ for $30 \mathrm{~min}$. FCM was performed using a FACSCalibur flow cytometer (Becton Dickinson). Data were analyzed by FlowJo software.

\section{Detection of tumor markers}

Levels of CEA, CA125, and CYFRA21-1 in serum of NSCLC patients were measured by full automatic electrochemiluminescent immunoassay (Roche, Switzerland). The upper limits of detection results were 3.5 $\mathrm{ng} / \mathrm{mL}, 35 \mathrm{U} / \mathrm{mL}$, and $3.3 \mathrm{ng} / \mathrm{mL}$, respectively.

\section{Graphing and statistical analysis}

Data were presented as Mean \pm SD. GraphPad Prism 5.0 (GraphPad, San Diego, CA) was used to generate bar graphs and ROC curves. The statistical significance of differences between groups was determined by the Student's t-test (two-tailed). Correlations between variables were determined by Spearman's correlation coefficient. Differences were considered significant at a $P$ value $<0.05$.

\section{RESULTS}

\section{CD15 ${ }^{+}$LOX-1 ${ }^{+}$PMN-MDSC frequency is increased in PB of NSCLC patients}

To compare the CD15+LOX-1+ PMN-MDSC frequency in NSCLC patients with that in healthy donors, we detected the proportion of $\mathrm{CD} 15^{+} \mathrm{LOX}-1^{+}$PMN-MDSCs in PB of the two groups. Detailed information of NSCLC patients were listed in Table 1. Based on the results of FCM, it was indicated that CD15+ LOX-1 ${ }^{+}$ PMN-MDSC frequency was significantly up-regulated in the circulation of NSCLC patients $(20.84 \pm 1.342 \%)$ compared to healthy individuals $(3.215 \pm 0.1346 \%$ ) (Figure $1, P<0.0001)$.

\section{$\mathrm{CD}^{+}{ }^{+}$LOX-1 ${ }^{+}$PMN-MDSC is a potential biomarker for NSCLC diagnosis}

To confirm the potential of CD $15^{+}$LOX-1 ${ }^{+}$PMN-MDSCs applied as a diagnostic marker for NSCLC, we firstly measured levels of CEA, CYFRA21-1 and CA125 in serum of NSCLC patients, and then analyzed their correlation with CD15 ${ }^{+} \mathrm{LOX}-1^{+} \mathrm{PMN}-\mathrm{MDSC}$ frequency. Results showed that levels of serum tumor markers including CEA $(7.348 \pm 1.024 \mathrm{ng} / \mathrm{mL})(P<0.0001)$, CYFRA21-1 $(7.436 \pm 0.9677 \mathrm{ng} / \mathrm{mL})(P<0.0001)$ and CA125 $(61.09 \pm 6.703 \mathrm{U} / \mathrm{mL})(P<0.0001)$ increased prominently in NSCLC patients compared with those in healthy donors (CEA: $1.77 \pm 0.1359 \mathrm{ng} / \mathrm{mL}$; CYFRA21-1: $1.799 \pm 0.1045 \mathrm{ng} / \mathrm{mL} ;$ CA125: $14.57 \pm 0.9361 \mathrm{U} / \mathrm{mL}$ ) (Figure 2A). We also found that CD $15^{+} \mathrm{LOX}-1^{+}$PMN-MDSC proportion was positively correlated with levels of CEA $(\mathrm{r}=0.6831, P=0.0021)$ and CYFRA21-1 $(\mathrm{r}=0.6598, P<0.0001)$, but not CA125 $(\mathrm{r}=0.2687, P$ $=0.0592$ ), in NSCLC patients (Figure 2B). Moreover, ROC curves of CD15 ${ }^{+} \mathrm{LOX}-1^{+}$PMN-MDSC frequency and tumor markers were analyzed. The results showed that AUC of PMN-MDSC frequency, CEA, CYFRA211 and CA125 in the diagnosis of NSCLC were 0.9996 (95\% CI: 0.9983-1.001), 0.8852 (95\% CI: 0.8226-0.9478), 0.9205 (95\% CI: 0.8670-0.9740) and 0.8209 (95\% CI: 0.7404-0.9015), respectively (Figure 2C). These results indicate that $\mathrm{CD} 15^{+} \mathrm{LOX}-1^{+}$PMN-MDSC is a potential biomarker for NSCLC diagnosis.

The proportion of $\mathrm{CD}^{+} 5^{+} \mathrm{LOX}-1^{+}$PMN-MDSCs is associated with NSCLC prognosis and recurrence after surgery

To confirm the connection between CD $15^{+}$LOX-1 ${ }^{+}$PMN-MDSCs and NSCLC prognosis, we detected the CD15 ${ }^{+}$LOX-1 ${ }^{+}$PMN-MDSC proportion in PB of NSCLC patients before and after surgical operation. The data showed that $\mathrm{CD} 15^{+} \mathrm{LOX}-1^{+}$PMN-MDSC frequency decreased significantly at one month after surgery 
$(20.84 \pm 1.3420$ vs $12.79 \pm 0.9396 \%, P=0.0058)$ (Figure 3A). Furthermore, we also measured the percentage of $\mathrm{CD} 15^{+} \mathrm{LOX}-1^{+}$PMN-MDSCs in NSCLC patients with or without recurrence after surgery. It was found that frequency of circulating CD $15^{+}$LOX $-1^{+}$PMN-MDSCs decreased significantly in NSCLC patients without recurrence compared to that in NSCLC patients with recurrence (13.76 \pm 0.9581 vs $19.91 \pm 0.7098$ $\%, P=0.0076$ ) (Figure 3B). In these cases, 11 samples could be traced from the NSCLC patients listed in

Table 1, in which there were 8 patients without recurrence and 3 patients with recurrence. By comparing the CD $15^{+}$LOX-1 ${ }^{+}$PMN-MDSC proportion in different groups, it was further confirmed that CD15 ${ }^{+}$LOX-1 $1^{+}$ PMN-MDSCs were related to NSCLC recurrence after surgery $(9.944 \pm 1.569$ vs $21.25 \pm 0.7963 \%, P<0.0001)$ (Figure 3C). All these data confirm that circulating CD15+LOX-1 ${ }^{+}$PMN-MDSC frequency is associated with NSCLC prognosis and recurrence after surgery.

\section{DISCUSSION}

Lung cancer is the leading cause of cancer-related death worldwide. As the main histological subtype of lung cancer, NSCLC attracts a lot of attention. In the past 20 years, advantages in the treatment of NSCLC have been achieved tremendously, and the understanding for NSCLC biology and progression has also been more comprehensive [8]. For instance, applications of small molecule tyrosine kinase inhibitors and immunotherapy have resulted in unprecedented survival benefits in selected patients [30-33]. However, the overall cure and survival rates of NSCLC patients still remain at a low rate, especially in metastatic condition [8]. MDSCs that are a population of IMCs with immunosuppression are a major cause of NSCLC immune escape, metastasis and therapy resistance [34-37]. As the major population of MDSCs, PMN-MDSCs account for 70-80\% of total MDSCs in both tumor-bearing mice and cancer patients. PMN-MDSCs suppress anti-tumor immune responses by releasing high levels of arginase 1 (ARG1) and reactive oxygen species (ROS) [38]. The phenotype of PMN-MDSCs is complicated. PMN-MDSCs were previously characterized as CD11b+ $\mathrm{Ly}^{+} \mathrm{G}^{+} \mathrm{Ly} \mathrm{C}^{\text {lowin }}$ mice, and were identified as $\mathrm{CD} 11 \mathrm{~b}^{+} \mathrm{CD} 33^{+} \mathrm{CD} 15^{+} \mathrm{CD} 66 \mathrm{~b}^{+} \mathrm{CD} 14^{-}$in human, which shared the same phenotype with mature neutrophils [15]. LOX-1 is a newly defined marker that can distinguish human PMN-MDSCs from mature neutrophils, and peripheral LOX-1 ${ }^{+}$PMN-MDSCs is associated with anti-PD-1 therapy in NSCLC patients [27]. Here, we demonstrated that CD15+LOX-1 $1^{+}$PMN-MDSC proportion increased in PB of NSCLC patients. The frequency of circulating CD15 ${ }^{+} \mathrm{LOX}-1^{+}$PMN-MDSCs was positively correlated with the levels of CEA and CYFRA21-1, and the combined detection of PMN-MDSCs and traditional tumor markers could enhance the diagnostic sensitivity and specificity for NSCLC. Moreover, we found that the proportion of $\mathrm{CD} 15^{+} \mathrm{LOX}-1^{+} \mathrm{PMN}-\mathrm{MDSCs}$ in PB of NSCLC patients decreased at one month after surgical resection, indicating that $\mathrm{CD} 15^{+} \mathrm{LOX}-1^{+}$PMN-MDSCs are associated with the prognosis of NSCLC. NSCLC recurrence after surgery is responsible for cancer associated mortalities after resection. To confirm the relationship between $\mathrm{CD} 15^{+} \mathrm{LOX}-1^{+}$PMN-MDSCs and NSCLC recurrence, we detected the PMN-MDSC frequency in NSCLC patients with or without recurrence after resection, and it was found that PMN-MDSC percentage was much higher in patients with recurrence. Therefore, CD $15^{+} \mathrm{LOX}-1^{+}$PMN-MDSCs are associated with NSCLC recurrence after surgery. However, PB samples in this study were all from primary NSCLC patients in early stage that were diagnosed for the first time and had not received any treatment before. CD $15^{+}$LOX-1 ${ }^{+}$PMN-MDSC presence in the circulation of III-IV NSCLC patients have not been revealed. Besides that, we only measured the frequency of CD $15^{+} \mathrm{LOX}-1^{+}$PMN-MDSCs before/after surgical treatment, but the relationship between $\mathrm{CD} 15^{+} \mathrm{LOX}-1^{+}$PMN-MDSCs and other treatments, such as radiotherapy, chemotherapy and targeted therapy, still needs further investigations.

\section{CONCLUSION}

In summary, CD15 ${ }^{+} \mathrm{LOX}-1^{+}$PMN-MDSC proportion increases in PB of NSCLC patients, and combined detection of PMN-MDSCs and traditional tumor markers can promote the diagnostic sensitivity and specificity for NSCLC. Moreover, CD15 ${ }^{+} \mathrm{LOX}-1^{+}$PMN-MDSCs are associated with NSCLC prognosis and recurrence after surgery.

\section{ACKNOWLEDGEMENTS:}

Not applied. 


\section{FUNDING:}

This work was supported by Jiangsu Planned Projects for Postdoctoral Research Funds (2018K253C), China's Post-doctoral Science Fund (2018ZM642225), the Fundamental Research Funds for the Central Universities (YG1805033+021414380392), and Funds of Nanjing Medical University Affiliated Cancer Hospital (ZM201704).

\section{DECLARATION OF INTEREST:}

No conflicts of interest.

\section{AUTHOR CONTRIBUTION:}

CONCEPTION: Xinyu Tian, Ting Wang

INTERPRETATION OR ANALYSIS OF DATA: Yue Tao, Qisi Zheng

PREPARATION OF THE MANUSCRIPT: Xinyu Tian, Han Shen

REVISION FOR IMPORTANT INTELLECTUAL CONTENT: Ting Wang

SUPERVISIN: Xinyu Tian, Ting Wang, Han Shen

All authors approved the final version.

\section{REFERENCES:}

1. Siegel RL, Miller KD, Jemal A. Cancer statistics, 2015. CA Cancer J Clin. 2015;65(1):5-29.

2. Torre LA, Bray F, Siegel RL, Ferlay J, Lortet-Tieulent J, Jemal A. Global cancer statistics, 2012. CA Cancer J Clin. 2015;65(2):87-108.

3. Oser MG, Niederst MJ, Sequist LV, Engelman JA. Transformation from non-small-cell lung cancer to small-cell lung cancer: molecular drivers and cells of origin. Lancet Oncol. 2015;16(4):e165-72.

4. Testa U, Castelli G, Pelosi E. Lung Cancers: Molecular Characterization, Clonal Heterogeneity and Evolution, and Cancer Stem Cells. Cancers (Basel). 2018;10(8).

5. Molina JR, Yang P, Cassivi SD, Schild SE, Adjei AA. Non-small cell lung cancer: epidemiology, risk factors, treatment, and survivorship. Mayo Clin Proc. 2008;83(5):584-94.

6. Hirsch FR, Scagliotti GV, Mulshine JL, et al. Lung cancer: current therapies and new targeted treatments. Lancet. 2017;389(10066):299-311.

7. Zugazagoitia J, Guedes C, Ponce S, Ferrer I, Molina-Pinelo S, Paz-Ares L. Current Challenges in Cancer Treatment. Clin Ther. 2016;38(7):1551-66.

8. Herbst RS, Morgensztern D, Boshoff C. The biology and management of non-small cell lung cancer. Nature. 2018;553(7689):446-54.

9. Pignon JP, Tribodet H, Scagliotti GV, et al. Lung adjuvant cisplatin evaluation: a pooled analysis by the LACE Collaborative Group. J Clin Oncol. 2008;26(21):3552-9.

10. Kris MG, Gaspar LE, Chaft JE, et al. Adjuvant Systemic Therapy and Adjuvant Radiation Therapy for Stage I to IIIA Completely Resected Non-Small-Cell Lung Cancers: American Society of Clinical Oncology/Cancer Care Ontario Clinical Practice Guideline Update. J Clin Oncol. 2017;35(25):2960-74.

11. Lu Z, Zou J, Li S, et al. Epigenetic therapy inhibits metastases by disrupting premetastatic niches. Nature. 2020;579(7798):284-90.

12. Iams WT, Porter J, Horn L. Immunotherapeutic approaches for small-cell lung cancer. Nat Rev Clin Oncol. 2020;17(5):300-12.

13. Forde PM, Chaft JE, Smith KN, et al. Neoadjuvant PD-1 Blockade in Resectable Lung Cancer. N Engl J Med. 2018;378(21):1976-86. 
14. Postmus PE, Kerr KM, Oudkerk M, et al. Early and locally advanced non-small-cell lung cancer (NSCLC): ESMO Clinical Practice Guidelines for diagnosis, treatment and follow-up. Ann Oncol. 2017;28 Suppl 4:iv1iv21.

15. Tian X, Shen H, Li Z, Wang T, Wang S. Tumor-derived exosomes, myeloid-derived suppressor cells, and tumor microenvironment. J Hematol Oncol. 2019;12(1):84.

16. Deng Z, Rong Y, Teng Y, et al. Exosomes miR-126a released from MDSC induced by DOX treatment promotes lung metastasis. Oncogene. 2017;36(5):639-51.

17. Bremnes RM, Busund LT, Kilvaer TL, et al. The Role of Tumor-Infiltrating Lymphocytes in Development, Progression, and Prognosis of Non-Small Cell Lung Cancer. J Thorac Oncol. 2016;11(6):789-800.

18. Caetano MS, Zhang H, Cumpian AM, et al. IL6 Blockade Reprograms the Lung Tumor Microenvironment to Limit the Development and Progression of K-ras-Mutant Lung Cancer. Cancer Res. 2016;76(11):3189-99.

19. Gabrilovich DI, Nagaraj S. Myeloid-derived suppressor cells as regulators of the immune system. Nat Rev Immunol. 2009;9(3):162-74.

20. Tian X, Ma J, Wang T, et al. Long non-coding RNA RUNXOR accelerates MDSC-mediated immunosuppression in lung cancer. BMC cancer. 2018;18(1):660.

21. Tian X, Ma J, Wang T, et al. Long Non-Coding RNA HOXA Transcript Antisense RNA Myeloid-Specific 1-HOXA1 Axis Downregulates the Immunosuppressive Activity of Myeloid-Derived Suppressor Cells in Lung Cancer. Front Immunol. 2018;9:473.

22. Tian J, Rui K, Tang X, et al. MicroRNA-9 Regulates the Differentiation and Function of Myeloid-Derived Suppressor Cells via Targeting Runx1. J Immunol. 2015;195(3):1301-11.

23. Tian X, Tian J, Tang X, et al. Particulate beta-glucan regulates the immunosuppression of granulocytic myeloid-derived suppressor cells by inhibiting NFIA expression. Oncoimmunology. 2015;4(9):e1038687.

24. Barrera L, Montes-Servin E, Hernandez-Martinez JM, et al. Levels of peripheral blood polymorphonuclear myeloid-derived suppressor cells and selected cytokines are potentially prognostic of disease progression for patients with non-small cell lung cancer. Cancer Immunol Immunother. 2018;67(9):1393-406.

25. de Goeje PL, Bezemer K, Heuvers ME, et al. Immunoglobulin-like transcript 3 is expressed by myeloidderived suppressor cells and correlates with survival in patients with non-small cell lung cancer. Oncoimmunology. 2015;4(7):e1014242.

26. Zhang Z, Huang X, Wang E, Huang Y, Yang R. Suppression of Mll1-Complex by Stat3/CebpbetaInduced miR-21a/21b/181b Maintains the Accumulation, Homeostasis, and Immunosuppressive Function of Polymorphonuclear Myeloid-Derived Suppressor Cells. J Immunol. 2020;204(12):3400-15.

27. Condamine T, Dominguez GA, Youn JI, et al. Lectin-type oxidized LDL receptor-1 distinguishes population of human polymorphonuclear myeloid-derived suppressor cells in cancer patients. Sci Immunol. $2016 ; 1(2)$.

28. Nan J, Xing YF, Hu B, et al. Endoplasmic reticulum stress induced LOX-1(+) CD15(+) polymorphonuclear myeloid-derived suppressor cells in hepatocellular carcinoma. Immunology. 2018;154(1):144-55.

29. Veglia F, Perego M, Gabrilovich D. Myeloid-derived suppressor cells coming of age. Nat Immunol. 2018;19(2):108-19.

30. Singh M, Jadhav HR. Targeting non-small cell lung cancer with small-molecule EGFR tyrosine kinase inhibitors. Drug Discov Today. 2018;23(3):745-53.

31. Cascone T, Morelli MP, Ciardiello F. Small molecule epidermal growth factor receptor (EGFR) tyrosine kinase inhibitors in non-small cell lung cancer. Ann Oncol. 2006;17 Suppl 2:ii46-8. 
32. Suresh K, Naidoo J, Lin CT, Danoff S. Immune Checkpoint Immunotherapy for Non-Small Cell Lung Cancer: Benefits and Pulmonary Toxicities. Chest. 2018;154(6):1416-23.

33. Osmani L, Askin F, Gabrielson E, Li QK. Current WHO guidelines and the critical role of immunohistochemical markers in the subclassification of non-small cell lung carcinoma (NSCLC): Moving from targeted therapy to immunotherapy. Semin Cancer Biol. 2018;52(Pt 1):103-9.

34. Dajon M, Iribarren K, Petitprez F, et al. Toll like receptor 7 expressed by malignant cells promotes tumor progression and metastasis through the recruitment of myeloid derived suppressor cells. Oncoimmunology. 2019;8(1):e1505174.

35. Li YD, Lamano JB, Lamano JB, et al. Tumor-induced peripheral immunosuppression promotes brain metastasis in patients with non-small cell lung cancer. Cancer Immunol Immunother. 2019;68(9):1501-13.

36. Zheng Y, Tian X, Wang T, et al. Long noncoding RNA Pvt1 regulates the immunosuppression activity of granulocytic myeloid-derived suppressor cells in tumor-bearing mice. Mol cancer. 2019;18(1):61.

37. Weber R, Fleming V, Hu X, et al. Myeloid-Derived Suppressor Cells Hinder the Anti-Cancer Activity of Immune Checkpoint Inhibitors. Front Immunol. 2018;9:1310.

38. Tian X, Zheng Y, Yin K, et al. LncRNA AK036396 Inhibits Maturation and Accelerates Immunosuppression of Polymorphonuclear Myeloid-Derived Suppressor Cells by Enhancing the Stability of Ficolin B. Cancer Immunol Res. 2020;8(4):565-77.

\section{Figure legends:}

Figure 1. CD $15^{+} \mathrm{LOX}-1^{+}$PMN-MDSC frequency in the circulation of healthy donors and NSCLC patients. The frequency of circulating CD $15^{+}$LOX $-1^{+}$PMN-MDSCs in healthy donors $(\mathrm{n}=50)$ and NSCLC patients $(\mathrm{n}=50)$ were measured by FCM. ${ }^{* * *} P<0.001$.

Figure 2. The potential application of CD $15^{+}$LOX-1 ${ }^{+}$PMN-MDSCs in NSCLC diagnosis. (A) Levels of CEA, CYFRA21-1, and CA125 in serum of NSCLC patients were measured $(\mathrm{n}=50)$. (B) The correlation of CD15 ${ }^{+}$LOX-1 ${ }^{+}$PMN-MDSC frequency with levels of tumor markers. (C) ROC curves. ${ }^{* * *} P<0.001$.

Figure 3. Circulating CD15 $15^{+} \mathrm{OX}-1^{+} \mathrm{PMN}-\mathrm{MDSCs}$ are associated with NSCLC prognosis and recurrence after surgery. (A) CD15+LOX-1 ${ }^{+}$PMN-MDSC frequency in PB of NSCLC patients before $(\mathrm{n}=50)$ or after surgical resection $(\mathrm{n}=50)$. (B) $\mathrm{CD} 15^{+} \mathrm{LOX}-1^{+} \mathrm{PMN}-\mathrm{MDSC}$ frequency in PB of NSCLC patients with $(\mathrm{n}=50)$ or without $(\mathrm{n}=50)$ recurrence post-surgery. (C) CD15 ${ }^{+} \mathrm{LOX}-1^{+}$PMN-MDSC frequency was compared in traced NSCLC patients with $(\mathrm{n}=8)$ or without $(\mathrm{n}=3)$ recurrence post-surgery. ${ }^{* *} P<0.01,{ }^{* * *} P<0.001$.

\section{Hosted file}

Table 1.pdf available at https://authorea.com/users/374114/articles/491729-circulating-cd15lox-1-pmn-mdsc-is-a-potential-biomarker-for-the-recurrence-after-surgery-of-non-smallcell-lung-cancer

\section{Hosted file}

Figures.pdf available at https://authorea.com/users/374114/articles/491729-circulating-cd15lox-1-pmn-mdsc-is-a-potential-biomarker-for-the-recurrence-after-surgery-of-non-smallcell-lung-cancer 